\title{
Kernos
}

Revue internationale et pluridisciplinaire de religion grecque antique

9 | 1996

Varia

\section{Hélène sur les vases attiques : esclave ou double d'Aphrodite}

\section{Christiane Bron}

\section{OpenEdition \\ Journals}

Édition électronique

URL : http://journals.openedition.org/kernos/1179

DOI : 10.4000/kernos. 1179

ISSN : 2034-7871

Éditeur

Centre international d'étude de la religion grecque antique

Édition imprimée

Date de publication : 1 janvier 1996

ISSN : 0776-3824

Référence électronique

Christiane Bron, « Hélène sur les vases attiques : esclave ou double d'Aphrodite », Kernos [En ligne], 9 | 1996, mis en ligne le 21 avril 2011, consulté le 22 avril 2019. URL : http://journals.openedition.org/ kernos/1179 ; DOI : 10.4000/kernos.1179 


\section{Hélène sur les vases attiques: esclave ou double d'Aphrodite}

Les sources littéraires, bien que primordiales pour notre compréhension des scènes peintes sur les vases attiques, ne sont souvent que des indices dirigeant l'interprétation : à Athènes l'image illustre rarement le texte. La transmission orale des mythes, toujours importante aux VIe et Ve siècles, semble plutôt dicter le choix des peintres de vases. Lorsqu'un épisode de la mythologie est représenté, des choix et des exclusions apparaissent, signes du sens que donnait à ses mythes la société athénienne. Ainsi, si l'on exclut quelques images de sa naissance et de son enlèvement par Thésée, le personnage d'Hélène n'intervient que dans deux scènes: l'enlèvement par Pâris, autour duquel viennent se greffer de multiples scènes de relations érotiques et de gynécée, et la reconquête par Ménélas. Aucune scène d'Hélène sur les murs de Troie, d'Hélène à proximité du cheval de bois ou en compagnie d'Ulysse, d'Hélène en Égypte ou d'eidolon d'Hélène ${ }^{1}$, alors que les textes, dramatiques surtout, font une large place à ce dernier épisode. Hormis quelques images de sa naissance, c'est donc Hélène épouse et amante que préfèrent les imagiers attiques.

\section{La jeune épousée}

Le personnage d'Hélène, belle jeune femme plus ou moins vêtue est difficile à identifier. Aucun attribut spécifique ne permet de la reconnaître et, souvent, une inscription est nécessaire pour diriger l'interprétation. Une des premières images du mariage d'Hélène se trouve sur un vase corinthien ${ }^{2}:$ le cortège nuptial répond au schème traditionnel des représentations à figures noires; il montre deux époux, Alexandros et Helena, montés sur un char; ils sont accompagnés des principaux héros troyens, tous nommés. À la même époque, un fragment de lébès attique, attribué à Sophilos, présente les noces d'Hélène et de Ménélas selon un schème identique ${ }^{3}$. Le cortège nuptial n'apparait que sur ces deux images archaïques.

\footnotetext{
1 Eur., Hél., 30-36; STÉS., 192-193 Davies.

2 Cratère corinthien, New York 27.116, L. GHALI-KAHIL, Les enlèvements et le retour d'Hélène, Paris, 1955, $\mathrm{n}^{\circ} 112$, pl 40, 1.

3 Lébès gamikos à figures noires, Izmir, $A B V$ 40, 20; GHALI-KAHIL, op. clt. (n. 2), $n^{\circ} 106$, pl. $87,1$.
} 
Vers la fin du VIe siècle, c'est le couple Hélène et Pâris ou Hélène et Ménélas qui est représenté, sans que l'identification des personnages puisse être assurée. Une allusion au mariage reste présente dans la posture de la femme : la tête couverte de son himation dont elle soulève un pan pour découvrir son visage elle reproduit le schème de la jeune mariée. Son compagnon, debout devant elle, l'entraîne en tenant son épée verticalement devant lui ou horizontalement en direction de la femme qui le suit. Cette différence iconographique permet d'interpréter l'image soit comme une représentation de l'enlèvement par Pâris, lorsque l'hoplite la précède sans la menacer (fig. 1) ${ }^{4}$, soit la reconquête par Ménélas, lorsque le guerrier dirige son épée contre elle (fig. 2) s $^{5}$ Enlevée ou agressée, Hélène apparaît sur le modèle de la jeune épousée qui suit docilement son amant ou son mari et cela jusqu'au dernier quart du Ve siècle.

Hélène partbénos ${ }^{6}, n y m p b \bar{e}^{-7}$, que sa beauté excuse et qui ne vieillit pas, Hélène à qui l'on peut attribuer une réinvention du mariage ${ }^{8}$, Hélène la déesse lacédémonienne qui, à Sparte, préside aussi bien à l'initiation des jeunes filles qu'à la consécration de leur beauté dans le mariage ${ }^{9}$ : toutes ces propriétés que les textes prêtent à l'héroïne, pourraient se trouver abrégées dans le choix de la représenter comme une jeune épouse, une nympbé, statut entre la partbénos, la vierge et la guné, la femme mariée ${ }^{10}$. Les mariages d'Hélène, avec Ménélas ou avec Pâris, ne sont que rarement mentionnés par les sources littéraires ${ }^{11}$; cette absence met en relief l'originalité de l'imagier et sa volonté de privilégier ce caractère nuptial d'Hélène.

4 Amphore à figures noires, Munich 1383, ABV 150, 7; GHALI-KAHIL, op.cit. (n. 2), n 6, pl. 1; $L I M C$, Hélène $\mathrm{n}^{\circ} 157, \mathrm{pl}$. 320. Ce type d'image est quelquefois interprétée comme les retrouvailles des fils de Thésée et d'Aithra.

5 Amphore à figures noires, Vatican, 16589, ABV 140,1; GHALI-KAHIL, op. cit. (n. 2), $\mathrm{n}^{\circ} 28$, pl 43, 2; LIMC, Hélène $\mathrm{n}^{\circ} 213, \mathrm{pl}$. 329. Les autres hommes, guerriers ou éphèbes qui entourent le couple central, deviennent alors les compagnons de Ménélas ou de Pâris, sans précision.

6 Voir P. VELKE, Beauté d'Hélène et rituels féminins dans l'Hélène d'Etripide, dans ce numéro de Kernos.

7 HOM., Il., III, 130; STÉS., 209 Davies.

8 Selon une interprétation des parties finales de trois catalogues de femmes (Hom., Od., XI, 436-439; SÉM. D'AMORGOS, fr. 7, 115 PT; Ehées, fr. 204, 97-112, M.-W.) proposée par Martin Steinrück lors de la réunion Coralie 1995 : le pas de transition entre l'âge hérö̈que et l'âge de fer, le présent, est marqué, dans l'esprit des Grecs, par un changement "biographique * entre l'âge des nonmariés et celui des mariés. Le fait de quitter Ménélas et de le retrouver, protection d'un ordre et d'un nouvel établissement, attribue à Hélène le rôle d'introduire le mariage à un niveau cosmologique.

9 C. CALAME, Les chceurs de jetunes filles en Grèce archaique, Rome, 1977, I, p. 333-350.

10 Pour la nymphé, voir M. DETIENNE, Le Mythe, Orphée au miel, in J. LE GOFF, P. NORA (éds), Faire de l'bistoire, Paris, 1974, III, p. 65-67; voir aussi CALAME, op. cit. (n. 9), p. 63 sq.

11 Selon GHALI-KAHIL, op. cit. (n. 2), p. 30, les Cypria mentionnent les noces à Troie. 


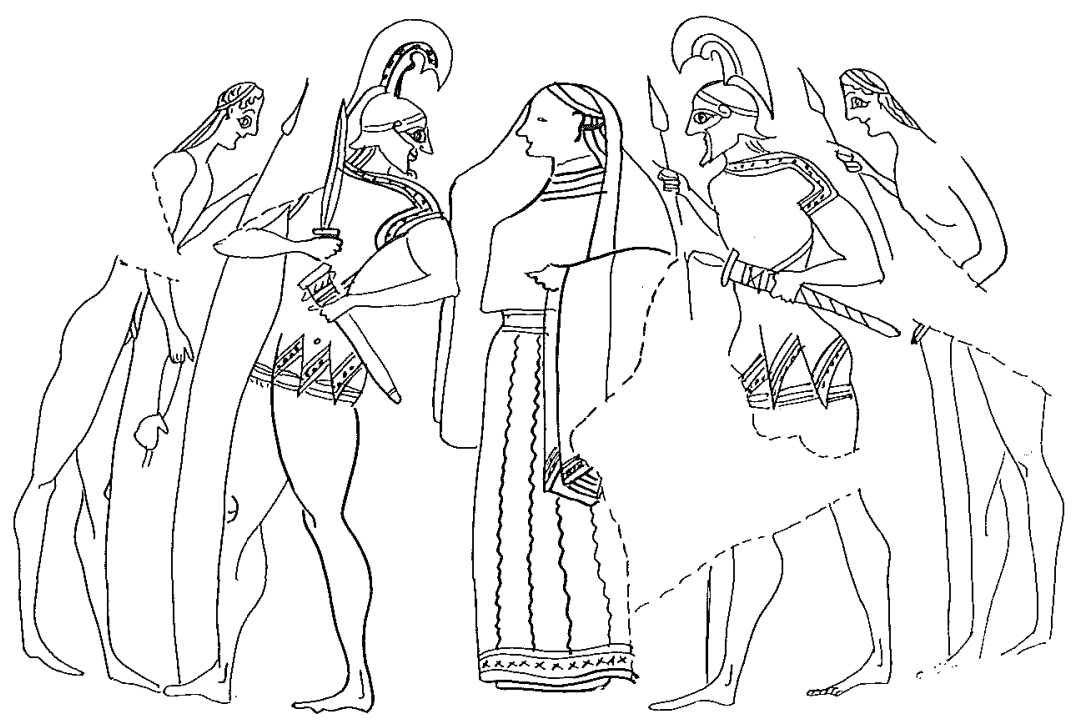

Fig. 1 : Amphore à figures noires, Munich 1383, $A B V 150,7$, dessin C. Bron.

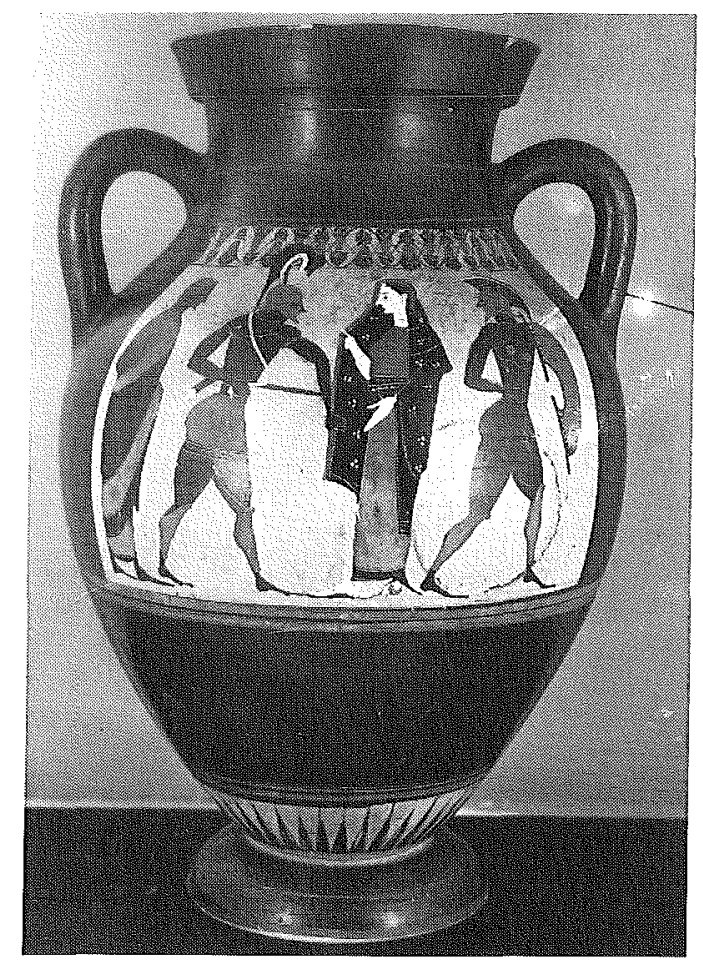

Fig. 2. : Amphore à figures noires, Vatican, 16589, $A B V 140,1$, photo du musée. 
Mariage, enlèvement, retrouvailles, l'ambiguïté des rapports de Pâris et de Ménélas avec Hélène est encore soulignée par une série d'images du début de la figure rouge. Le jeune guerrier qui précède une femme et la tient par le poignet répond au schème de Pâris entraînant Hélène. Il faut remarquer que cela ne correspond pas vraiment à un enlèvement ${ }^{12}$, mais bien plutôt à une relation amoureuse, une prise de possession de la fiancée. C'est le geste de l'époux conduisant sa jeune femme dans les scènes de mariage dès le dèbut du Ve siècle ${ }^{13}$. C'est aussi le modèle qu'adopte Macron sur le célèbre skyphos du musée de Boston ${ }^{14}$ (fig. 3); Éros qui survole le couple précise encore cette relation amoureuse.

Pourtant sur un lécythe de Berlin, le même éphèbe, coiffé d'un casque, muni d'une lance, est nommé Ménélas par une inscription (fig. 4$)^{15}$. La jeune femme qui le suit, la tête couverte de son himation, le regard baissé reproduit le schème de la jeune mariée des vases à figures rouges ${ }^{16}$, comme nous l'avons déjà mentionné. Elle a d'ailleurs la même attitude que l'Hélène, enlevée par Pâris du skyphos de Boston, (fig. 3). Une jeune épousée précédée d'un éphèbe nommé Ménélas il pourrait donc s'agir du mariage d'Hélène et de Ménélas.

Pourtant l'époux n'est jamais représenté en armes et le geste de saisir la fiancée par le poignet peut aussi bien évoquer le moment où Ménélas retrouve Hélène, à Troie, que leurs noces à Sparte. Le casque de hoplite porté par Pâris se justifie par le côté séditieux de son acte, pour Ménélas, le port d'un vêtement guerrier ne s'explique pas lors de ses noces à Sparte. Malgré l'inscription cette image reste ambiguë et souligne le caractère nuptial d'Hélène. L'interprétation de l'épisode des retrouvailles à Troie est généralement déterminée par la présence d'une épée nue dont le guerrier, presque toujours barbu, menace la jeune femme qui le suit. Pourtant, même en présence de l'épée, Hélène garde l'attitude de la fiancée suivant docilement son amant ou son mari (fig. 5) ${ }^{17}$. Peut-on voir dans ces images une allusion au "remariage » d'Hélène et de Ménélas?

12 L'enlèvement d'Hélène par Thésée est rarement représenté sur les vases attiques. Dans ce cas, il s'agit vraiment d'un enlèvement : la jeune fille est portée à bras le corps par un Thésée qui s'enfuit vers le char conduit par Pirithoos; GHALI-KAHIL, op. cit. (n. 2), pl. 102-104.

13 Sur la signification de ce geste, voir S.H. LONSDALE, Dance and Ritual Play in Greek Religion, Baltimore, 1993, p. 214. C'est également le geste de la danse « nuptiale » des garçons et des filles qui décore le bouclier d'Achille : HOM., Il, XVIII, 594. Voir aussi J.H. OAKLEY, R.H. SINOS, The Greek Wedding in Ancient Atbens, Wisconsin Univ. Press, 1993, p. 32.

14 Skyphos à figures rouges, Boston 13.186, ARV 458, 1; GHALI-KAHIL, op.cit. (n. 2), $\mathrm{n}^{\circ}$ 11, pl. 4; LIMC, Hélène $\mathrm{n}^{\circ} 166$, pl. 321.

15 Lécythe à figures rouges, Berlin F 2205, ARV 383, 202; GHALI-KAHIL, op. cit. (n. 2), $\mathrm{n}^{\circ} 107$, pl. 85,2 ; LIMC, Hélène $n^{\circ} 62$, pl. 302. Image unique de Ménélas imberbe, sans inscription on $y$ reconnaîtrait obligatoirement Pâris.

16 Voir F. LISSARRAGUe, Femmes au figuré, in G. DUBY, M. PERroT (éds), Histolre des femmes, l'Antiquité, Paris, 1991, I, p. 163-184; OAKLEY et SINOS, op. clt. (n. 13), passim.

17 Plat, Odessa 0.577, ARV 67, 137; GHALI-KAHIL, op. cit. (n. 2), nº 99, pl. 82, 2; LIMC, Hélène $\mathrm{n}^{\circ} 310, \mathrm{pl} .348$. 


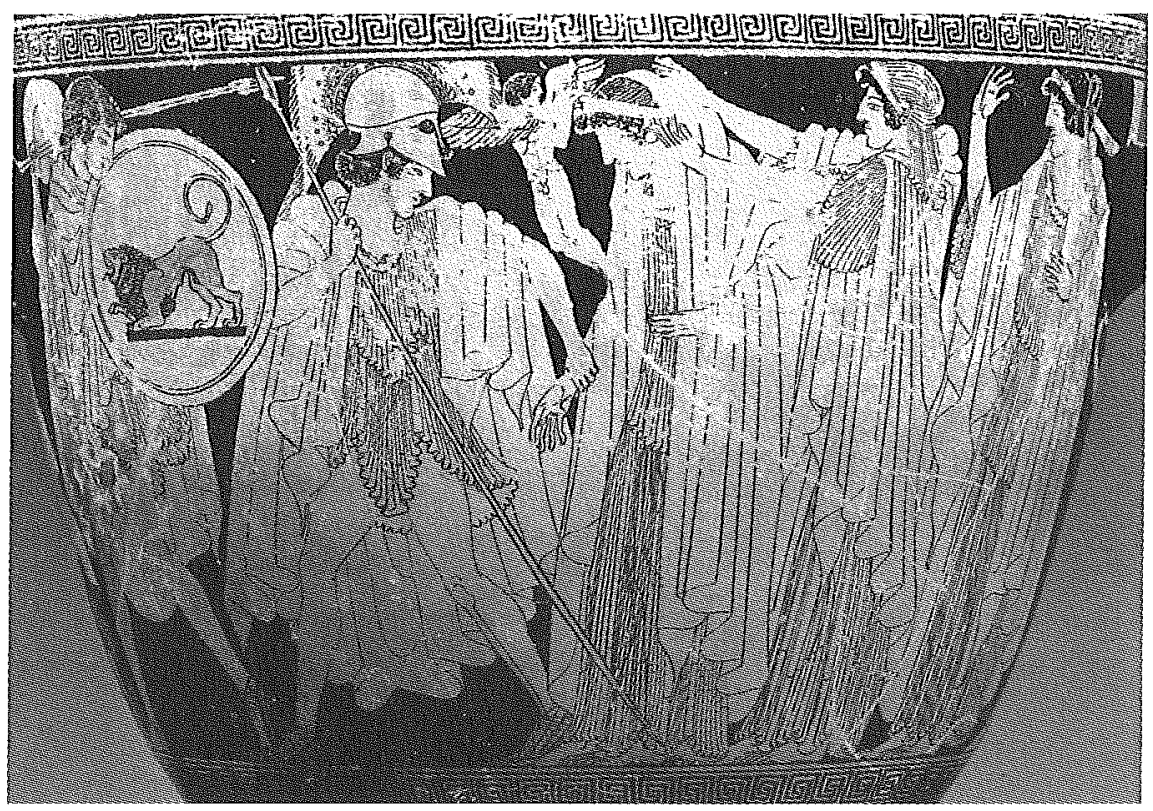

Fig. 3. : Skyphos à figures rouges, Boston 13.186, $A R V 458,1$, photo du musée.

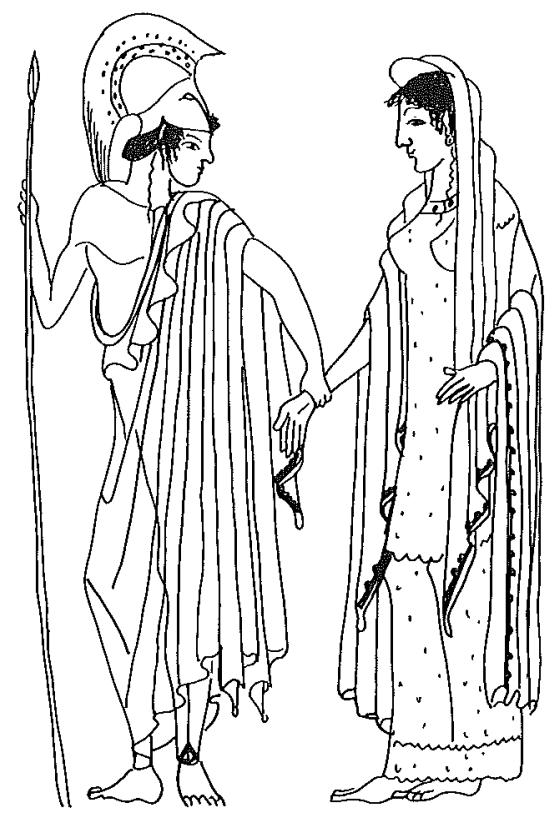

Fig. 4 : Lécythe à figures rouges, Berlin F 2205, ARV 383, 202, dessin C. Bron 


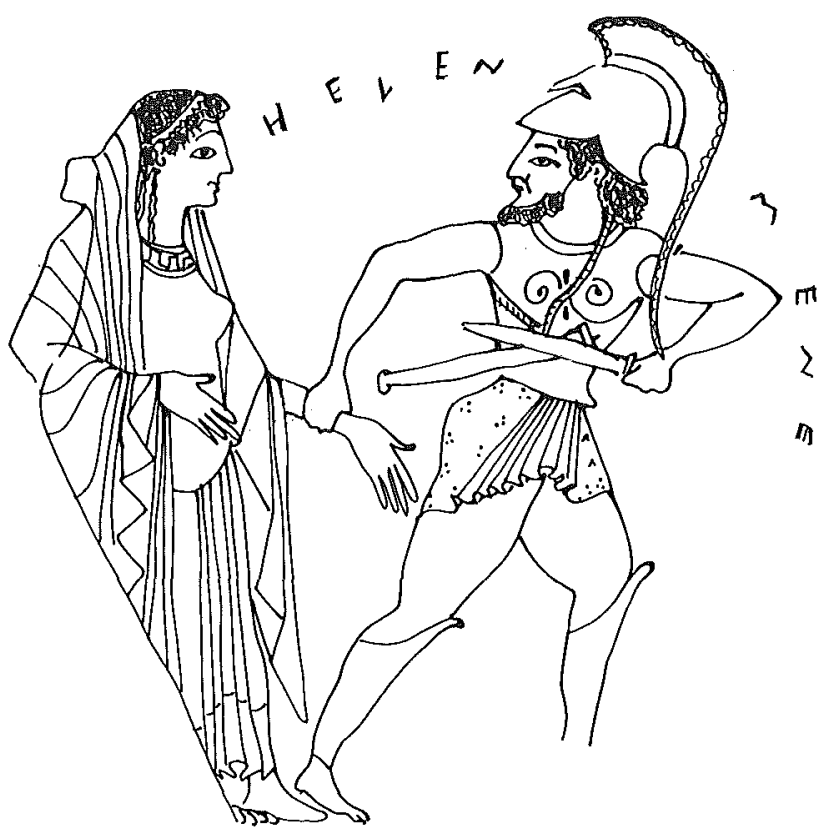

Fig. 5 : Plat à figures rouges, Odessa $0.577, A R V 67,137$, dessin C. Bron.

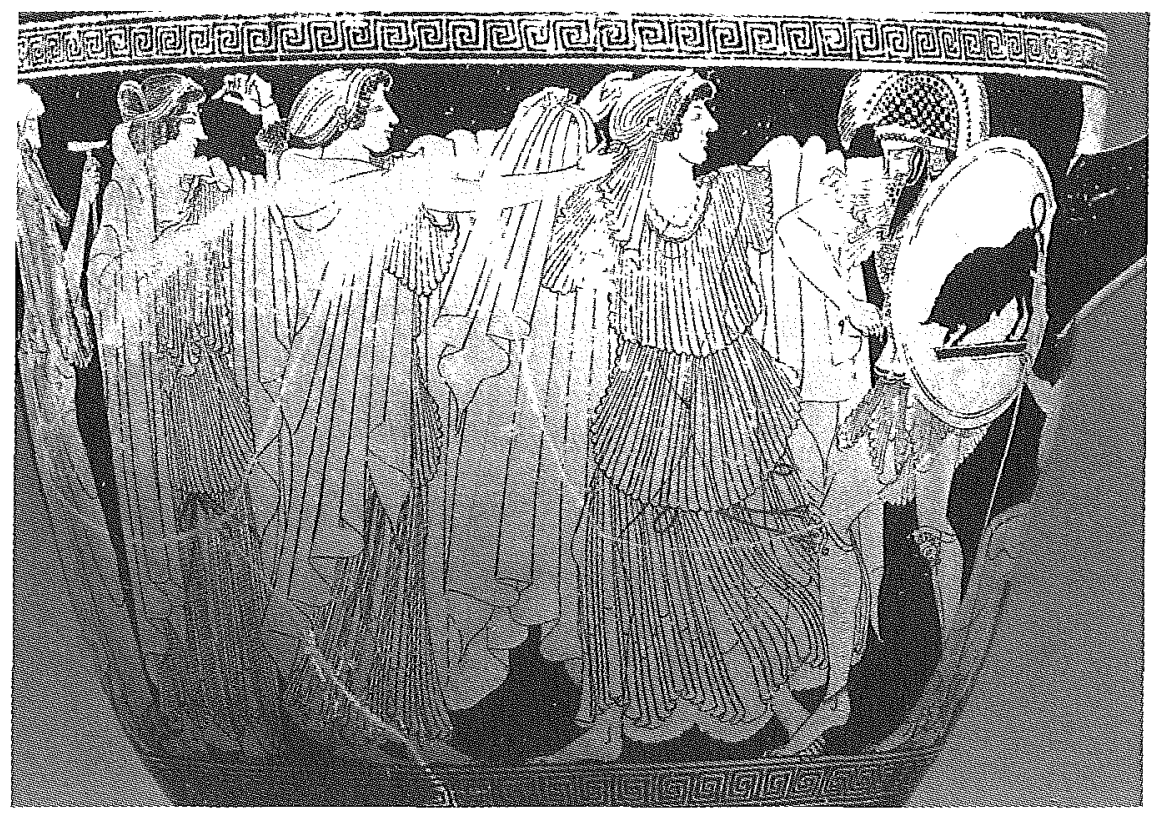

Fig. 6. : Skyphos à figures rouges, Boston 13.186, $A R V 458,1$, photo du musée (= fig. 3). 


\section{Esclave d'Aphrodite}

Ce n'est qu'au début du Ve siècle que le schème éclate et que les deux épisodes sont clairement différenciés. C'est alors qu'Aphrodite apparaît, déesse de la beauté et de l'amour : elle préside au destin d'Hélène, elle l'a promise à Pâris et elle intervient de toute sa puissance pour réaliser le vœu du Troyen. Le skyphos de Macron résume les deux rôles d'Hélène : celui de la fiancée qui suit docilement son ravisseur (fig. 3) et celui de l'épouse qui s'enfuit devant son mari jaloux (fig. 6) ${ }^{18}$. Aphrodite intervient : secondée de Peitho (la persuasion ${ }^{19}$ et d'Éros, lorsqu'elle préside à l'enlèvement d'Hélène (fig. 3) ou prête à recueillir Hélène dans ses bras lorsqu'elle la protège de la colère de Ménélas, armé de pied en cap (fig. 6). Ce schème de la fuite d'Hélène annonce celui de la poursuite amoureuse qui sera repris sur de nombreux vases tout au long du Ve siècle. Poursuivant et poursuivie peuvent avoir des identifications très diverses qui sont généralement précisées par l'adjonction de signes iconiques caractéristiques des héros ou des dieux mis en scène ${ }^{20}$.

Ménélas se reconnaît à son costume hoplitique et souvent à la présence d'Aphrodite et d'Éros, quelquefois de Peitho. Mais ce qui le distingue à coup sûr des autres poursuivants c'est l'épée qui tombe de sa main, marquant ainsi son émotion devant la beauté de sa femme (fig. 7 ) $^{21}$. Lorsqu'elle est présente, Aphrodite souligne sa protection et la dépendance d'Hélène à son égard par un geste de la main, et par l'envoi d'Éros qui vole au-devant de l'époux jaloux, marquant ainsi l'efficacité de la puissance divine de la déesse de l'amour. C'est elle aussi qui provoque l'émotion de Ménélas, la renaissance de son amour et l'abandon de sa volonté d'agression. Responsable de la fuite et de l'adultère de la reine, elle se doit d'empêcher le mari jaloux de malmener son épouse. Dans ces images, plus ou moins contemporaines de l'Hélène d'Euripide, l'épisode de son séjour en Égypte et l'eidolon, son double troyen ${ }^{22}$, n'apparaissent pas. Comme Gorgias, les peintres invoquent le pouvoir d'Aphrodite, l'impuissance de l'homme devant les menées divines pour disculper Hélène ${ }^{23}$. Cette dépen-

18 Revers du vase cité supra, n. 14, GHAli-KAHIL, op. cit. (n. 2), nº 53 pl. 48; LIMC, Hélène $n^{\circ} 243$, pl. 334 .

19 Sur Peitho, personnification et déesse de la persuasion voir H.A. SHAPIRO, Personifications in Greek Art, Zürich, 1993, p. 186-207.

20 Sur le schème de la poursuite érotique voir C. SOURVINOU INWOOD, Menace and Pursuit: Differentiation and the Creation of Meaning, in C. BÉRARD et al. (éds), Images et Société en Grèce Ancienne, l'iconograpbie comme métbode d'analyse, Lausanne 1987, p. 40-55; ainsi que, du même auteur, A series of Erotic Pursuits: Images and Meanings, in JHS, 107 (1987) p. 131-153; voir aussi S. KAEMPF-DIMITRIADOU, Die Liebe der Götter in der Attiscben Kunst des 5. Jabrbunderts v. Cbr., Berne, 1979, passim.

21 Entre autres cratère à figures rouges, Paris G 424, ARV 1077, 5; GHALI-KAHIL, op. cit. (n. 2), $\mathrm{n}^{\circ}$ 66, pl. 63, 3; LIMC, Hélène $\mathrm{n}^{\circ}$ 268; voir aussi l'œenochoé, Vatican 16535, ARV 1173; GHALI-KAHIL, op. cit. (n. 2), $\mathrm{n}^{\circ} 72$, pl. 64, 1-3; LIMC, Hélène $\mathrm{n}^{\circ} 272$ bis, pl. 340.

22 EUR, Hél., 30-36; S'TÉs., 192-193 Davies.

23

GORG., Hêl., 6. 
dance d'Hélène à l'égard d'Aphrodite est également très présente dans les scènes de l'enlèvement ou de la rencontre avec Pâris : Éros, Aphrodite et souvent Peitho unissent leurs efforts pour la convaincre de suivre le prince troyen (fig. 3).

Le caractère inéluctable du destin d'Hélène, jouet entre les mains des dieux, est particulièrement évident sur un amphoriskos de Berlin (fig. 8) ${ }^{24}$. Hélène, sur les genoux d'Aphrodite ${ }^{25}$, garde l'attitude de la fiancée, la tête inclinée, couverte de son himation. Derrière elle, à gauche, Peitho, la persuasion, Tyché (?), la fortune et Némésis, la vengeance; devant elle, Himeros, le désir qui attire Pâris, Heimarméné, la déesse du destin et une dernière jeune femme anonyme qui tient un oiseau ${ }^{26}$. Ainsi sont réunies autour de l'héroïne toutes les puissances qui président à son destin, avec au centre la déesse de la Beauté, cause de l'infidélité, de la guerre et des destructions futures. Hélène est impuissante, petite fiancée fragile que l'on rassure, elle n'est que l'esclave de la volonté divine. En cela les peintres de vases rejoignent les sources littéraires, Hélène n'est pas fautive : c'est le destin, le plan des dieux qui's'exécute à travers elle ${ }^{27}$.

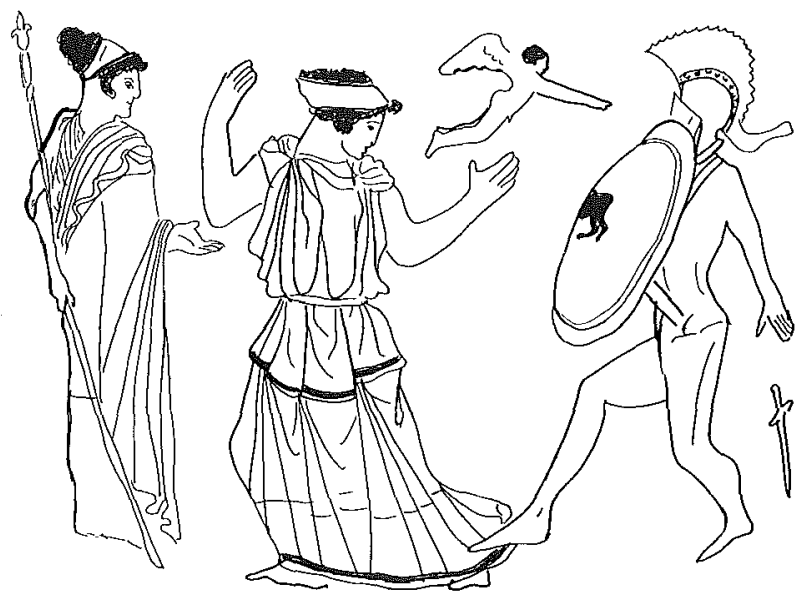

Fig. 7 : Cratère à figures rouges, Paris G 424, $A R V 1077,5$, dessin C. Bron.

24 Amphoriskos à figures rouges, Berlin 30036, ARV 1173, 1; GHALI-KAHIL, op. cit. (n. 2), $\mathrm{n}^{\circ} 14$, pl. 8, 2-3; LIMC, Hélène $\mathrm{n}^{\circ} 140, \mathrm{pl} 316$.

25 Ce geste se retrouve entre autres dans le cercle du peintre de Meidias, voir le lécythe d'Athènes, 6471, $A R V 1175,11$, interprété comme une image d'Aphrodite, LIMC, Aphrodite $\mathrm{n}^{\circ} 210$, pl. 24 et sur une hydrie de Florence, 81948, ARV 1312, 1.

26 Pour une analyse plus détaillée de ce vase, voir H.A. SHAPIRO, The Origins of Allegory in Greek Art, in Boreas, 9 (1986) p. 9 sq.

27 HOM., Il., III, 160-165; GORG., Hél., 6; SAPPHO, fr. 16 LP/Voigt. 


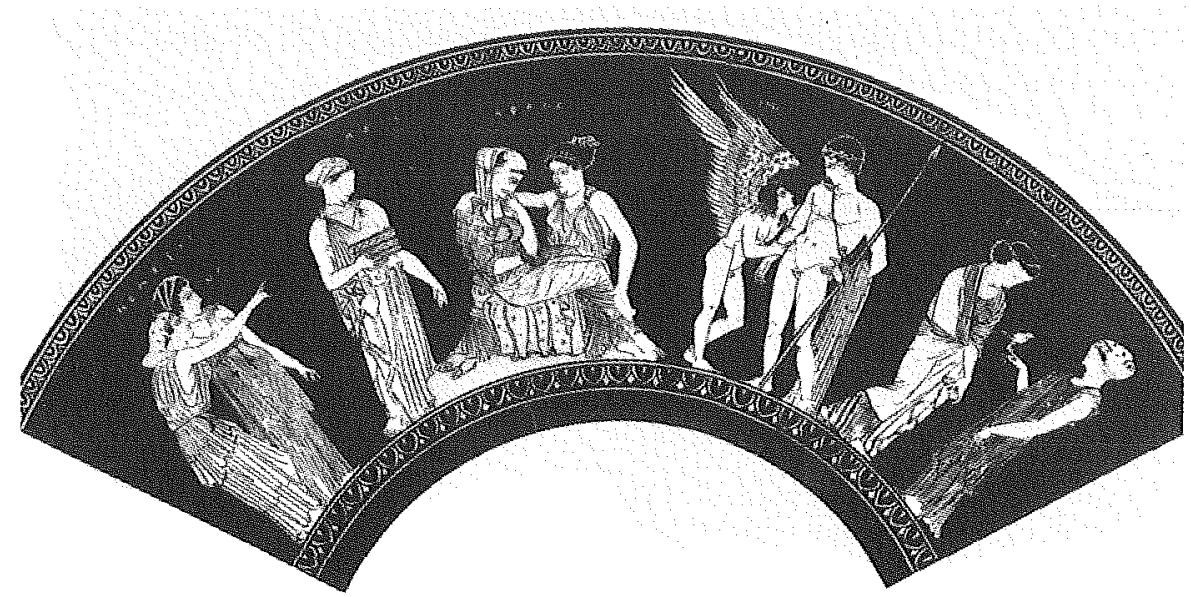

Fig. 8 : Amphoriskos à figures rouges, Berlin 30036, $A R V 1173,1$, photo tirée de L. Ghali-Kahil, $n^{\circ} 14$, pl. 8, 2-3.

\section{Double d'Aphrodite}

Au tournant du Ve et du IVe siècles, Hélène n'est plus la jeune fiancée timide ou l'épouse soumise; elle revendique son caractère de "plus belle femme du monde ». Ménélas, rappel de la faute d'Hélène, disparaît des images. Ce sont la relation amoureuse de Pâris et Hélène et la beauté d'Hélène qui sont représentées sur les vases attiques. Évolution d'un goût esthétique, témoignage d'un changement de mentalité, ce choix est corroboré par les textes. Isocrate dans son Éloge d'Hélène considère la Beauté comme un don de Zeus qu'il réserve à sa fille; c'est le pouvoir suprême qui supplante tous les autres, même la force ${ }^{28}$; c'est aussi le plus précieux des dons, celui que même les déesses recherchent ${ }^{29}$. L'amour de Pâris et d'Hélène est glorifié, l'Amour comme la Beauté sont des puissances auquelles l'homme ne peut résister ${ }^{30}$.

Le départ avec Pâris, lorsqu'il est représenté, tient plus de l'apothéose que de l'enlèvement. Sur un lécythe de Saint Pétersbourg, les deux amants sont environnés d'Erotes (fig. 9) ${ }^{31}$ : Hélène sur le char, se présente comme une

28 ISOCR., Hél., 16.

29 ISOCR., Hél., 48 et 54 .

30 GORG., Hél., 18-20; ISOCR., Hél., 42 et 48

31 Lécythe aryballistique de Saint-Pétersbourg St 1929, GHALI-KAHIL, op. cit. (n. 2), $\mathrm{n}^{\circ} 159$, pl. 6, 1; $L I M C$, Hélène $\mathrm{n}^{\circ} 172, \mathrm{pl} 322$. Cette représentation d'Hélène, réplique d'Aphrodite n'est pas unique, voir entre autres l'cuf de New York, $\mathrm{n}^{\circ}$ 1971.258.3, ARV 1256, 1; GHALI-KAHIL, op. cit. (n. 2), $\mathrm{n}^{\circ}$ 23, pl. pl. 5; LIMC, Hélène $\mathrm{n}^{\circ}$ 171, pl 322, sur lequel Hélène et Aphrodite portent le même vêtement et le même diadème, l'une est sur le char aux côtés de Pâris, l'autre les regarde partir, en compagnie d'Éros. 
réplique d'Aphrodite, debout devant les chevaux. Toutes les deux sont nues, coiffées de façon identique ${ }^{32}$. Pâris en prince oriental conduit l'attelage, Hermès est devant et les Dioscures derrière.

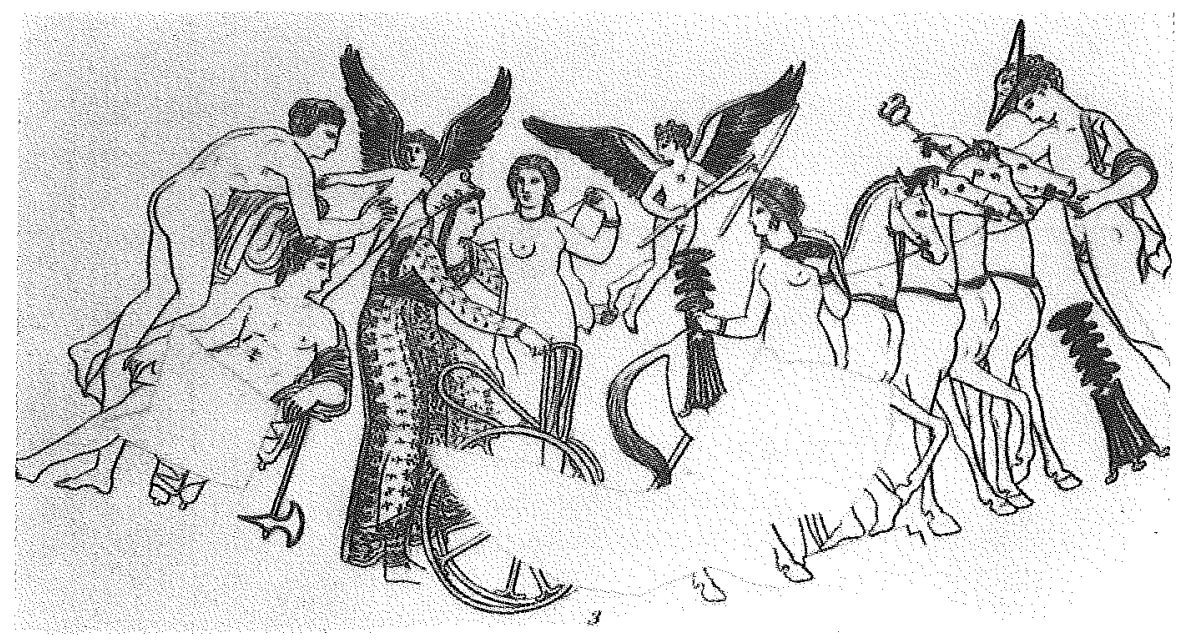

Fig. 9 : Lécythe aryballistique de Saint-Pétersbourg St 1929, dessin d'après L. Ghali-Kahil, n 159 , pl. 6, 1.

À cette même époque, les scènes de gynécée qui, souvent, font allusion au mariage mais aussi au pouvoir d'Aphrodite, deviennent très courantes ${ }^{33}$. Les femmes entourent la fiancée ${ }^{34}$, assise au centre, en lui offrant coffrets, paniers, couronnes et rubans. Lorsque des Erotes volètent alentour, il n'est pas toujours aisé de préciser si la femme ainsi honorée est Hélène (fig. 10) ${ }^{35}$, Aphrodite ${ }^{36}$, ou une fiancée humaine ${ }^{37}$. Le jeu des offrandes ${ }^{38}$, la position de l'Éros ou le recours à l'inscription peuvent orienter l'interprétation.

32 Sur le Beauté qui rend Hélène immortelle, qui l'amène au rang des dieux, voir IsocR., Hél., $60-61$.

33 LissarRague, art. cit. (n. 16), p. 174 sq.

34 Voir entre autres : les loutrophores de Paris N 3406, ARV 1320, 3 et d'Oxford 1927.4067, ARV 1179, ainsi que le lébès gamikos de Paris CA 1861.

35 Hydrie Londres E 226, $A R V$ 1318, 3; GHALI-KAHIL, op. cit. (n. 2), n 124, pl. 28, 3; LIMC, Hélène $\mathrm{n}^{\circ} 78, \mathrm{pl} 305$.

36 Lécythe Londres E 697, ARV 1324, 45; LIMC, Aphrodite $\mathrm{n}^{\circ} 1271$, pl 127.

37 Sur l'importance d'Aphrodite dans les jeux de séduction, voir HOM., Il., XIV, 198-224.

38 La présence d'offrande de coffrets pourrait faire glisser l'interprétation du côté d'une scène humaine, mais la vérification de cette hypothèse dépasse le cadre de ce travail. 


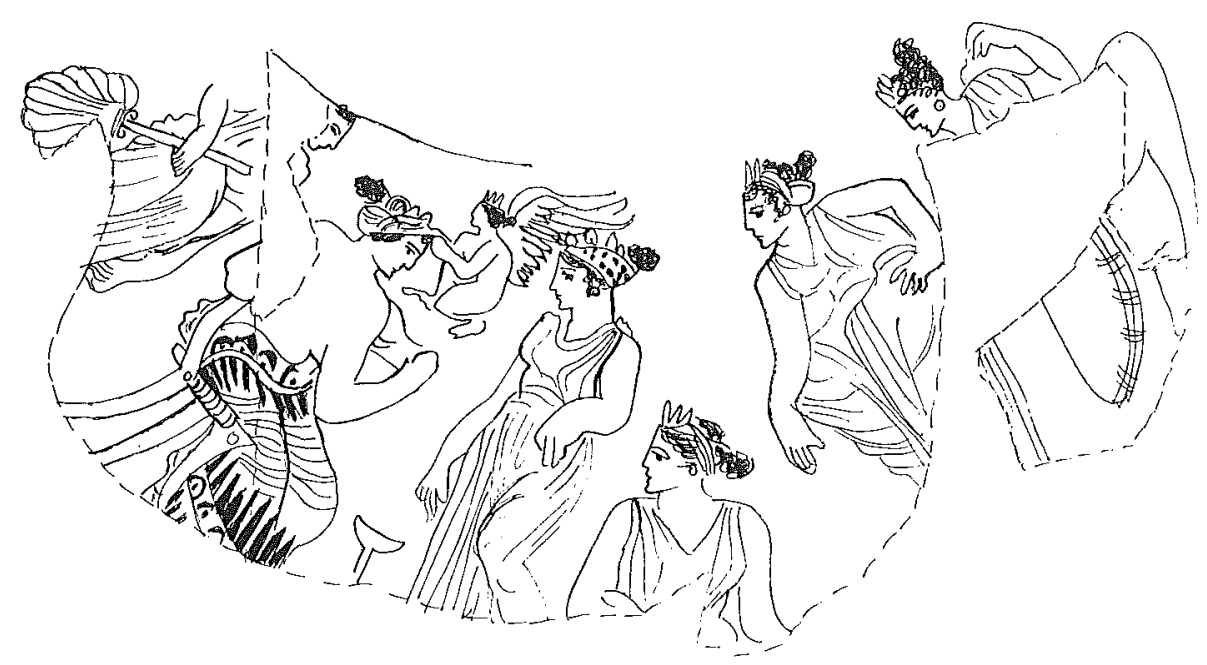

Fig. 10 : Hydrie Londres E 226, $A R V 1318,3$, dessin C. Bron.

Lorsque qu'Aphrodite ou Hélène sont au centre de l'image, les suivantes sont souvent des allégories féminines ${ }^{39}$ qu'une inscription permet d'identifier. Ainsi sur l'hydrie de Londres (fig. 10), Kalé - la beauté - en buste et Kléo - la renommée - debout derrière elle sont à droite d'Hélène; Pothos - le désir est accroupi devant le visage d'Hélène. La présence de Kléo, symbole de la renommée, de la gloire, rappelle les paroles d'Hélène dans l'Iliade ${ }^{40}$ : dans son dialogue avec Hector, elle précise qu'elle restera dans les mémoires parce qu'elle sera chantée par les poètes ${ }^{41}$. Le présence de Kleo donne donc à l'image une dimension de véhicule glorifiant du mythe au même titre que le poème. Hélène apparaît à nouveau dans un rôle de fiancée mais aussi dans celui de double d'Aphrodite, surtout lorsqu'elle dévoile sa beauté, beauté soulignée par le nom Kalé donné à l'une de ses suivantes. Sa renommée est ici uniquement due à sa beauté, ni Pâris, ni Ménélas n'interviennent dans ce chemin vers l'immortalité.

Hélène dont «la beauté achevée est égale à celle d'Aphrodite ${ }^{42}$ apparaît encore sur les vases selon le modèle de l'Aphrodite accroupie, l'Aphrodite à sa toilette, schème qui sera repris tout au long de l'antiquité sous

39 Sur les personnifications sur les vases attiques, voir SHAPIRO, op. cit. (n. 19), passim.

40 HOM., Il., VI, 357-358.

41 Sur les liens entre Hélène et le kleos, la gloire, dans une analyse de l'Hélène d'Euripide, voir G.S. MELTZER, * Where is the Glory of Troy?. Kleos in Euripides' Helen, in Clant, 13 (1994), p. 234-255.

42 CALAME, op. cit. (n. 9), I, p. 344 : 
diverses formes : statues, relief, fresque et intailles ${ }^{43}$. Sur un lécythe de la collection Embiricos ${ }^{44}$ (fig. 11) : Hélène nue, accroupie, se lave les cheveux avec l'aide d'un Éros; Pothos et Aphrodite à droite la regardent tandis qu'Eukleia et peut-être Peitho (inscription incomplète) leur font pendant sur la gauche. Eukleia porte dans ses mains la couronne, reprenant le thème de la renommée cité ci-dessus, alors qu'Aphrodite, munie d'un sceptre, est représentée dans toute sa gloire.
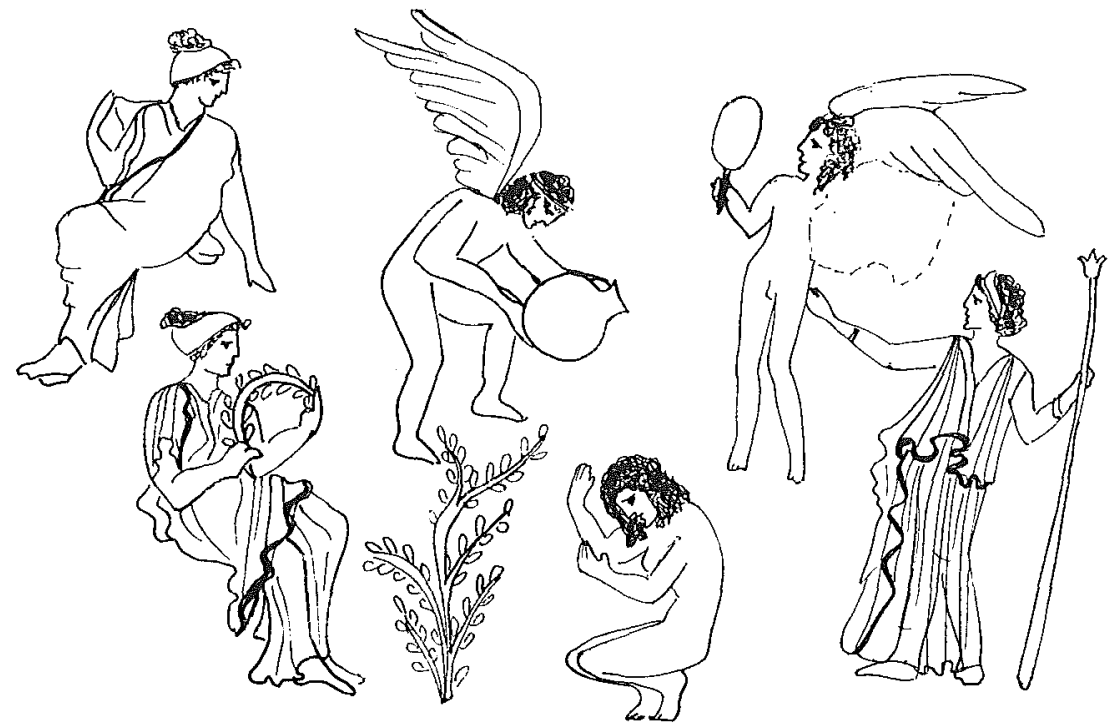

Fig. 11 : Lécythe, coll. Embiricos, ARV 1690, 6ter, dessin C. Bron.

Une jeune femme presque identique, entourée d'Erotes se retrouve sur un lécythe de Berlin ${ }^{45}$ et sur un épinetron de Rhodes ${ }^{46}$, image de cette Aphrodite accroupie, contemporaine du lécythe Embiricos (fig. 11). Le bain, la purification par l'eau font aussi allusion au rituel du mariage ${ }^{47}$. Symbole de l'acceptation de l'hymen, cette eau sera celle que les Danaïdes porteront éternellement vers un récipient sans fond, expiant ainsi le refus du mariage et le meurtre des fils

43 Voir D. BRINKERHOFF, Hypothesis on the History of the Crouching Apbrodite Type in Antiquity, in The J. Paul Getty Museum Joumal, 6-7 (1978-1979), p. 83-96, fig. 11. Il hésite à voir dans la jeune femme du lécythe de Berlin (infia, n. 45) une Artémis, à cause du faon qui se trouve devant elle, mais la nudité et la présence des deux Éros font pencher l'interprétation vers Aphrodite. Voir les Aphrodites de ce type, LIMC, Aphrodite $n^{\circ}$ 985-1042, p. 96-104.

44 Lécythe, coll. Embiricos, ARV 1690, 6ter; LIMC, Hélène n 77, pl. 305.

45 Lécythe Berlin 2707, ARV 1326, 70; LIMC, Aphrodite n 988, pl. 96.

46 Épinétron à figures rouges Rhodes 13886, ARV 1503, 6; LIMC, Aphrodite $\mathrm{n}^{\circ}$ 990, pl. 96.

47 R. GINoUvÈs, Balaneuttké, Paris, 1962, chap. 3, Le bain et les rites du mariage.. 
d'Égyptos ${ }^{48}$. Peut-on voir dans la représentation d'Hélène au bain une nouvelle allusion à l'ambiguîté du rôle qui lui est assigné : déesse du passage entre la jeune fille virginale parthénos et la jeune mariée nymphé, objet du désir par les artifices d'Aphrodite?

\section{Conclusion}

Les liens d'Hélène et d'Aphrodite sont donc très clairement représentés sur les vases attiques, pourtant c'est en tant que fiancée et jeune épousée que se présente l'héroïne d'Homère, trois fois mariée ${ }^{49}$ ! Ce n'est pas l'image de la reine de Sparte, de la femme adultère, responsable des massacres de Troie, mais une nymphé, une jeune fille que l'on prépare ou que l'on mène à ses noces. Son rôle de déesse présidant à " l'achèvement de la beauté et à la maturité $e^{50} \gg$ des jeunes filles avant le mariage est peut-être symbolisé par cette représentation des peintres attiques. Mais c'est surtout à Sparte que ce culte est attesté, en relation directe avec les cultes à Aphrodite.

En Attique, les textes parlent d'Hélène, ils excusent son adultère au nom du destin fixé par les dieux, de la puissance de sa Beauté et de l'Amour ${ }^{51}$, ils le nient en créant un eidolon ${ }^{52}$, son double parfait, envoyé par les dieux pour la remplacer en ville de Troie. Parallèlement, les imagiers présentent Hélène comme une héroïne, jouet des dieux qui accomplit leur dessein ou image terrestre d'une égale des déesses immortelles, comme le soulignent les anciens de Troie en la regardant passer ${ }^{53}$.

Par ces représentations, les imagiers attiques témoignent d'une inspiration voisine des textes de Gorgias ou d'Isocrate, reflet d'un état d'esprit qui tend peut-être à réinterpréter le mythe selon de nouveaux critères éthiques. Ils marquent peut-être un écart avec Euripide, qui présente Hélène comme une parthénos, ainsi que le montre l'étude de $\mathrm{P}$. Vœlke, «Beauté d'Hélène et rituels féminins dans l'Hélène d'Euripide ${ }^{54} \%$. La beauté d'Hélène signifie un prolongement anormal du statut de parthénos mais aussi de celui de nymphé. ${ }^{-55}$ L'image ne nous permet pas de juger de la valeur négative ou positive de sa

J.-P- DARMON, Puissances du mariage, in Dictionnaire des mytbologies et des religions des sociétés traditionnelles et du monde antique, Paris, 1981, II, p. 69-71 s.v. mariage.

49 Épouse de Ménélas, de Pâris et de Déiphobos.

50 CALAME, op. cit. (n. 9), II, p. 12.

51 GORG., Hél., 21; ISOCR, Hél., 48.

52 EUR., Hél., 30-36; STÉs., fr. 192-193 Davies.

53 HOM., Il., II, 156.

54 VGELKE, op, cit. (n. 6).

55 C'est aussi le statut de Pénélope, mariée et mère qui reste une njmphé en l'absence d'Ulysse, mais qui retrouve son statut de gyné à son retour, voir I. PAPADOPOULOU-BELMEHDI, Le chant de Pénélope. Poétlque du tissage fëminin dans l'Odyssée, Paris, 1994, p. 105-107 et 126-127. 
beauté, mais elle recoupe clairement les témoignages littéraires et présente Hélène comme une éternelle nymphé malgré son statut de femme mariée et de mère.

Institut d'Archéologie et d'Histoire Ancienne

Christiane BRON Université de Lausanne

BFSH II

CH - 1015 DORIGNY 